\title{
A randomized control trial: Effects of teach-back method on self-efficacy among mothers of children with congenital heart defects
}

\author{
Azza A. Ghoneim, Amal AbdElrazk Fathalla \\ Department of Pediatric Nursing, Faculty of Nursing, Menoufia University, Menoufia, Egypt
}

Received: November 26, 2017

DOI: $10.5430 /$ jnep.v8n7p106
Accepted: February 8, $2018 \quad$ Online Published: March 14, 2018

URL: https://doi.org/10.5430/jnep.v8n7p106

\begin{abstract}
Background/Objective: Congenital Heart Defects (CHD) remain a major health concern all over the world particularly Egypt where the prevalence of CHD is 1.0 per 1,000. Nurses are instrumental in supplying information. The teach-back method is a technique used for improving patient understanding and outcomes. This study aimed to evaluate the effect of teach-back method on self-efficacy and satisfaction among mothers of children with congenital heart defects.

Methods: The design of this study was randomized control trial. A sample of 60 children with congenital heart defects and their mothers participated in this study. It conducted at Menofia University hospital. Tools of this study included Self Efficacy Scale; Teach back Discharge Education Audit and Satisfaction Assessment.

Results: The current study revealed that the majority of nurses were unfamiliar with teach-back method and there was a significant difference between mothers in the experimental and control groups regarding their self-efficacy.

Conclusions: This study concluded that mothers who received discharge instructions through teach-back method had increased self-efficacy and high level of satisfaction. Therefore, pediatric nurses should integrate teach back method as a routine nursing intervention in the discharge plan for children with congenital heart defects.
\end{abstract}

Key Words: Congenital heart defects, Teach-back method, Self-efficacy

\section{INTRODUCTION}

Congenital Heart Defects (CHD) are the second cause of death in infancy and childhood. ${ }^{[1]}$ A congenital heart defect is an abnormal heart structure present at birth. ${ }^{[2]}$ The exact cause of CHD is unknown, however; there are environmental and genetic risk factors or a combination of both. ${ }^{[3]}$ The most frequent risk factors that result in CHD are rubella infection during pregnancy, alcohol and tobacco administration, poor nutrition and genetic abnormalities such as Down syndrome. ${ }^{[4-6]}$
Signs of CHD are varied and related to the type and severity of the defect. Some children have no signs meanwhile; others exhibit poor feeding, cyanosis, dyspnea and murmur and growth retardation. ${ }^{[2]}$ Children with CHD are vulnerable to developing congestive heart failure, stroke, chest infection, therefore, they require a lifelong follow-up and care. ${ }^{[7]}$ Frequent hospitalization and treatment modalities of CHD create a great burden on parents particularly mothers that may impair their self-efficacy. Self-efficacy is the certainty that persons can successfully perform a particular behavior

${ }^{*}$ Correspondence: Azza A. Ghoneim; Email: azza.ghonaim@nursing.menofia.edu.eg; Address: Department of Pediatric Nursing, Faculty of Nursing, Menoufia University, Menoufia, Egypt. 
and expect the results. ${ }^{[8]}$

Self-efficacy is the mediator between individual's knowledge and action. It used as a model for evaluating healthpromoting education in cardiac rehabilitation and adherence to prescribed regimens. ${ }^{\left[{ }^{[3]}\right.}$ Consequently, self-efficacy is an essential key to determining and influencing health outcomes. Children with CHD and their parents need knowledge and information about the effective ways of taking care of their defects. ${ }^{[10]}$

Effective management of CHD include parents understanding of the health condition of their child, monitoring symptoms, incorporating diet recommendations; adhering to medications, following activity restrictions, follow-up appointment and knowing when to seek medical advice. ${ }^{[11]}$ Consequently, those families will need verbal and written instructions. Nurses have a tremendous responsibility in instructing and educating patients. ${ }^{[12]}$

Patient education is a vital aspect of nursing care and may consider the core of nursing practice. Effective education can improve health, reduce hospital readmissions, decrease the costs of healthcare, and improve patient and family satisfaction. ${ }^{[12]}$ Therefore, nurses should utilize effective and feasible techniques to assure patient comprehension of instructions. ${ }^{[13]}$

Teach-back method is a technique, whereby patient/caregiver should convey the received information from a provider and clarifying the feedback. It reflects patient's comprehension of the instructions intended to be thought. ${ }^{[14]}$ Teach-back method is relying on the basis in cognitive psychology that repeating short sequences of information helps to improve recall of information. ${ }^{[15]}$ Actually, at discharged, the parent often does not know what medications the physicians have prescribed, what diet and activity restrictions and when the follow-up appointments should take place. Therefore, teach-back will increases patients adherence to disease management throughout enhancing patients' knowledge and selfmanagement skills. ${ }^{[11]}$

Although, the teach-back technique is recommended as a 'universal precaution' by the Agency for Healthcare Research and Quality (AHRQ) and the National Quality Forum, ${ }^{[16-18]}$ there are few studies evaluating the effects of teach-back method on self-efficacy among mothers of children with congenital heart defects. Therefore, the study was conducted.

\subsection{Aim of the study}

This study aimed to evaluate the effect of teach-back method on self-efficacy among mothers of children with congenital heart disease.

Published by Sciedu Press

\subsection{Hypothesis}

Mothers of children with CHD who would receive discharge instruction through teach-back method would have more selfefficacy and satisfaction than mothers who would receive discharge instruction through regular method.

\section{MeTHODS}

\subsection{Research design}

This study utilized a randomized control trial design.

\subsection{Settings}

This study conducted in the pediatric department at Menofia University hospital in Shebin El-Kom city.

\subsection{Subjects}

A simple random sample of 60 children and their mothers were divided into two equal groups that were control and experimental. In addition, 40 nurses working at pediatric units at Menoufia University hospital. Sample size (n) was estimated based on the formula: $\mathrm{n}=\bar{p}(1-\bar{p}) \times\left(\mathrm{z}_{1-\alpha / 2}\right.$ $\left.+\mathrm{z}_{1-\beta}\right)^{2} \div\left(\mathrm{p}_{1}-\mathrm{p}_{2}\right)^{2}$ with a confidence level of 0.95 and test power $80 \%$. Where $\mathrm{n}$ is the sample size, $\bar{p}=\left(\mathrm{p}_{1}+\mathrm{p}_{2}\right)$ $\div 2, \mathrm{p}_{1}=0.50, \mathrm{p}_{2}=0.34$. The estimated sample size was 74 participants; however, 14 children were excluded due to referral to cardiac surgery and children's death.

\subsection{Inclusion criteria}

Children were included in the study according to the following criteria:

(1) Children diagnosed with congenital heart disease.

(2) Free from other chronic diseases to prevent further stress on mothers.

\subsection{Instruments}

The researchers utilized four tools to collect the data. These tools included:

Tool I: Structured Interviewing Questionnaire. The researcher developed it. It composed of two parts:

Part one: Characteristics data. It contained items related to age, gender, mothers' education, occupation, nurses' level of education and age.

Part two: Teach-back method assessment sheet. It contained three statements that assess nurses' familiarity with teachback method.

Tool II: Self-Efficacy Scale to assess the general self-efficacy of mothers caring for children with congenital heart disease. It adopted from Sherer \& Madox work. ${ }^{[19]}$ It included 17 questions rated on a five-point scale in which items ranging from (5) completely agree to (1) completely disagree. 
Scoring system included the sum of item scores. The high the total score is, the high the self-efficacy Total score of self-efficacy scale is 85 . The level of self-efficacy considered high if the score was $>42-85$ and low if the score was 0-42. Reliability of the tool was $r=0.85$.

Tool III: Teach-back Discharge Education Audit, Sullivan ${ }^{[20]}$ developed it. Reliability of the tool was determined by Cronbach's co-efficiency alpha test. It was $r=0.68$. It composed of two parts:

Part one: Assessment of Nurses' teach-back skills. It is an observational checklist. It contained six statements to assess nurses' skills of implementing teach-back method during discharge (e.g., nurse-initiated mother's understanding of procedure done, the nurse used eye contact, short phrases, layman's terms, nurse provide time for mother questions).

Part two: Assessment of retained instructions. It composed of four questions to assess patient retention of discharge instruction (e.g., mother able to describe the activity limitations, describe the medication name and dose, state dietary recommendations and state the location, date and time of next follow up visit).

Tool IV: Mother's satisfaction of teach-back method. It included two statements indicating mother's satisfaction regarding retention of discharge instructions before and after utilization of teach-back method. Scoring items ranged from unsatisfied (1), sometimes satisfied (2) to satisfied (3).

\subsection{Validity}

Tools developed and adopted by the researcher for data collection after searching of relevant literature. For validity assurance purpose, tools submitted to a jury of five experts in the field of pediatrics and pediatric nursing.

\subsection{Pilot study}

In order to ascertain that the tools of the study are clear, feasible and applicable, a pilot study carried out on 10 mothers and 10 nurses. No modifications made. Therefore, they were included in the study.

\subsection{Ethical considerations}

For ethical considerations, an oral consent was obtained from nurses and mothers at Menofia University hospital to participate in the study. Therefore, the objectives of the study, its importance, safety and confidentiality were clarified.

\subsection{Procedure}

Data collection starting from Jun 2015 to January 2016. Data collected throughout three periods: on child admission, during discharge and during follow up visit. Data collection process guided by phases of Lippitt's theory of change.

\subsection{Theoretical framework}

Change theory by Lippitt's attributed the theoretical base for the current study. Lippitt assumed that change is comprised of six phases: diagnose the problem, assess individual's motivation and capacity for change, assess change agents' motivation and resources, choose objective that is progressive, choose appropriate role of change agent, maintain change once it has been initiated. ${ }^{[21]}$ These phases related to the elements of nursing process that includes assessment to identify the problem, planning for an appropriate intervention, implementation of the required change and evaluation of its effect. These phases promote effective implementation of the teach-back method that contributes to making the desirable change.

\section{A. Pre-intervention phase}

(1) The researchers developed the time plan for conducting the proposed change throughout four phases that were pre-intervention, in-service, post-intervention and follow-up.

(2) The researchers meant to gather the baseline data from nurses and from mothers. Therefore, an interview with nurses working at pediatric inpatient word, pediatric clinic and pediatric intensive care unit conducted to assess their knowledge and practice regarding teach-back method.

(3) In addition, assessing mothers' retention of discharge instruction by the routine discharge process. It included questions on the instruction of medication dose and time, diet and activity restrictions, weight measurement, prevention of chest infection, next echo appointment and next follow up appointment.

(4) Then the researcher assessed mothers' self-efficacy.

(5) Therefore, the researcher developed a PowerPoint presentation, brochure and handouts on teach-back method guidelines for nurses.

\section{B. In-service phase}

(1) The researcher clarified the findings of the preintervention assessment. Nurses recognized the advantages of the teach-back method in discharge planning and they were willing to participate in the study.

(2) The researcher divided nurses into four equal groups (10 nurses per group) and conducted four educational sessions each lasted for 30 to 45 minutes. These sessions included definition; benefits and technique of teach back method. Teaching strategies were group discussion, role-play and workshop.

\section{Post intervention phase}

Upon completion of educational sessions, the researcher re-assessed nurses' knowledge regarding teach-back and 
re-assessed mothers' retention of discharge instruction and value $<.05$ for all significant statistical tests.

mothers' self-efficacy and satisfaction.

\section{Follow up phase}

The researcher assessed mothers' self-efficacy during follow up visit. It often was within one week after discharge for most of the children.

\subsection{Data analysis}

Researchers coded, transformed and entered the collected data into a designed form then analyzed by using the Statistical Package of the Social Scientists (SPSS) Version 22.0. Quantitative data analyzed by mean $(\bar{X})$ and standard deviation (SD). Student $t$-test was utilized to compare the two means. Qualitative data was presented in the form of number and percentage. It was analyzed by chi-square $\left(\chi^{2}\right)$ test. If an expected value of any cell in the table was less than 5 , Fisher Exact test was used. Level of significance was set at $p$

\section{Results}

Table 1 shows characteristics of experimental and control groups. It clarified that more than half of children (66.7\%; $56.7 \%$ ) in experimental and control groups were female. There were no statistically significant differences between children in experimental and control groups regarding their mean age ( $4.7 ; 9.1$ respectively).

Figure 1 illustrated familiarity with teach-back method among nurses. It clarified that the majority of nurses (96.6\%; 93.3\%) in experimental and control groups were unfamiliar with the teach-back method for discharge planning.

Figure 2 showed mothers' comprehension to discharge instructions at pre-intervention phase. It clarified that the more than half of mothers $(65 \%)$ in were unable to describe the discharge instruction clearly.

Table 1. Characteristics of participants in the experimental and control groups

\begin{tabular}{|c|c|c|c|c|c|}
\hline \multirow{2}{*}{ Characteristics } & \multicolumn{2}{|c|}{ Experimental group $(n=30)$} & \multicolumn{2}{|c|}{ Control group $(n=30)$} & \multirow{2}{*}{$t$-test $/ \chi^{2}$} \\
\hline & $\%$ & No & $\%$ & No & \\
\hline \multicolumn{6}{|l|}{ Sex } \\
\hline Male & 33.3 & 10 & 43.3 & 13 & $0.6^{\mathrm{ns}}$ \\
\hline Female & 66.7 & 20 & 56.7 & 17 & \\
\hline Children age & Mean \pm SD & $4.7 \pm 8.9$ & $9.1 \pm 5$ & & $0.14^{\mathrm{ns}}$ \\
\hline Mother's age & Mean \pm SD & $31.57 \pm 2.99$ & $31.2 \pm 2.07$ & & $0.55^{\mathrm{ns}}$ \\
\hline
\end{tabular}

Note. ${ }^{\text {ns }}$ not significant

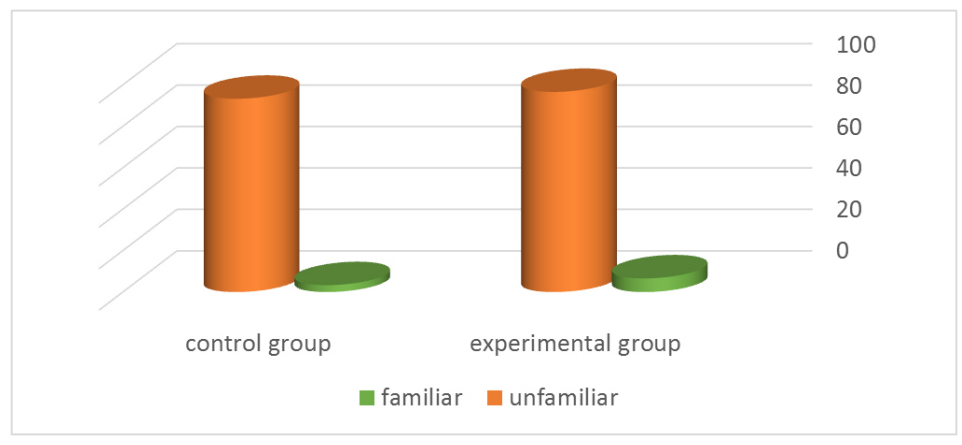

Figure 1. Familiarity with teach-back method among nurses

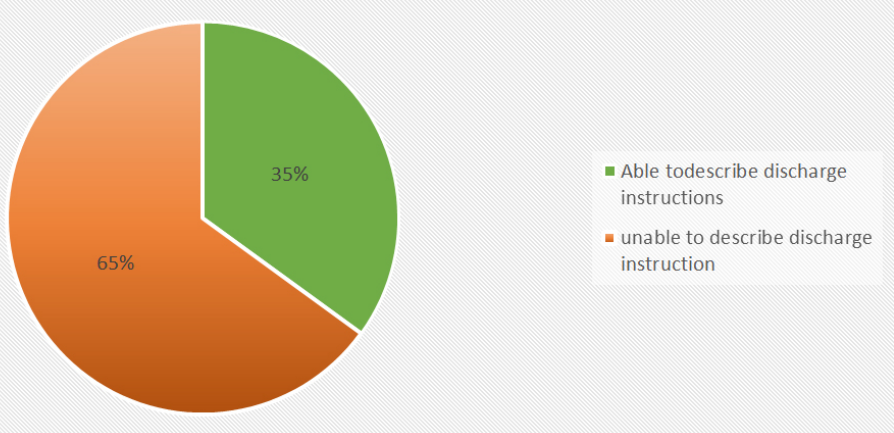

Figure 2. Mothers' comprehension to discharge instruction at pre-intervention phase 
Table 2 shows Self-efficacy of mothers in the experimental group at pre and post teach back. It clarified that self-efficacy of mothers in the experimental group was improved at postintervention and follow up phases $(57.1 \pm 10.3,52.4 \pm 8.4)$ respectively. Therefore, there were high statistical significant differences between experimental and control groups at post-intervention and follow up phase.

Table 2. Self-efficacy of mothers in the experimental group at pre and post intervention phase

\begin{tabular}{|c|c|c|c|c|}
\hline \multirow[b]{2}{*}{ Phase } & \multicolumn{2}{|l|}{ Self- efficacy } & \multirow[b]{2}{*}{$t$-test } & \multirow[b]{2}{*}{$p$} \\
\hline & $\begin{array}{l}\text { Experimental group }(n=30) \\
(\text { Mean } \pm \text { SD) }\end{array}$ & $\begin{array}{l}\text { Control group }(n=30) \\
(\text { Mean } \pm \text { SD) }\end{array}$ & & \\
\hline Pre-intervention & $37.7 \pm 6.8$ & $39.7 \pm 8.3$ & 1.99 & $.38^{\mathrm{ns}}$ \\
\hline Post-intervention & $57.1 \pm 10.3$ & $39.6 \pm 8.4$ & 15.67 & $.001^{* *}$ \\
\hline 1st follow up visit & $52.4 \pm 8.4$ & $40.6 \pm 8.5$ & 10.48 & $.001^{* *}$ \\
\hline $\mathrm{F}$ & 114.11 & 10.12 & & \\
\hline
\end{tabular}

Figure 3 shows assessment of discharge instructions among mothers in experimental and control groups at postintervention. It clarified that the majority of mothers in the experimental group $(96.6 \%)$ at post-intervention phase were clearly describe the discharge instruction, meanwhile, the majority of mothers in the control group (93.3\%) were unable to describe it. Therefore, there was statistical significant difference between mothers regarding retaining of discharge instructions.

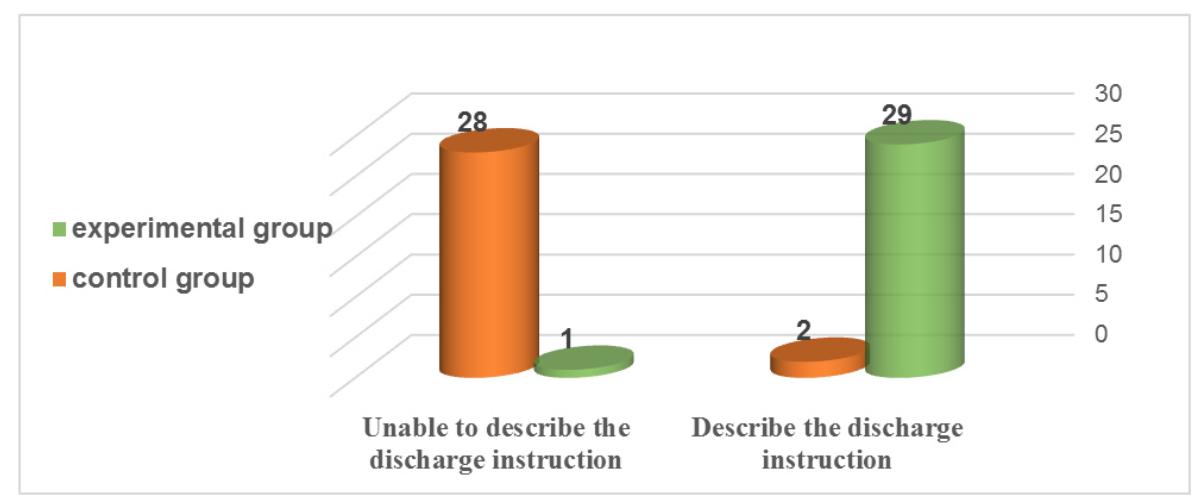

Figure 3. Assessment of discharge instructions among mothers in experimental and control groups at post-intervention

Table 3 shows mother's level of satisfaction in the experimental group at pre and post teach-back method. It clarified that more than half of mothers in the experimental group were very satisfied $(60 \%)$ and about one quarter $(26.7 \%)$ were slightly satisfied at post-intervention phase. Therefore, there was statistically significant difference between mothers at pre- and post-intervention phase regarding their satisfaction with teach-back method.

Table 3. Mother's level of satisfaction in the experimental group at pre and post teach-back method

\begin{tabular}{|c|c|c|c|c|c|c|}
\hline \multirow{3}{*}{ Satisfaction Level } & \multicolumn{4}{|c|}{ Experimental group } & \multirow{3}{*}{ Total } & \multirow{3}{*}{$\begin{array}{l}\chi^{2} \\
p\end{array}$} \\
\hline & \multicolumn{2}{|c|}{ Pre-intervention $(n=30)$} & \multicolumn{2}{|c|}{ Post-intervention $(n=30)$} & & \\
\hline & $\mathbf{N}$ & $\%$ & $\mathbf{N}$ & $\%$ & & \\
\hline Satisfied & 0 & 0 & 18 & 60 & 18 & \multirow{3}{*}{$\begin{array}{l}16.364 \\
.000^{*}\end{array}$} \\
\hline Sometimes satisfied & 4 & 13.3 & 8 & 26.7 & 12 & \\
\hline Un satisfied & 26 & 82.7 & 4 & 13.3 & 30 & \\
\hline
\end{tabular}




\section{Discussion}

Congenital heart disease is a significant health problem in children that could be life-threatening. Researchers indicated that about $30 \%-50 \%$ of childhood mortality from birth defects are relying on CHD. ${ }^{[22]}$ Frequent hospitalization is common among children with CHD and ideally, discharge planning begins on admission. ${ }^{[23]}$ Clear discharge instructions and successful education to children with congenital heart disease is the key of their health. Patient education is a core competency of nursing practice that improves health outcomes and reduces hospital readmissions. ${ }^{[23]}$ Therefore, nurses should utilize innovated and effective teaching method in order to reinforce mothers' understanding of discharge instruction.

The current study hypothesized that mothers of children with CHD who would receive discharge instruction through teach-back method would have more self-efficacy and satisfaction than mothers who would receive discharge instruction through regular method.

Regarding nurses' familiarity with teach-back method at pre-intervention phase, the present study revealed that the majority of nurses $(96.6 \%$; $93.3 \%)$ in the experimental and control groups were unfamiliar with the teach-back method for discharge planning. These findings are inconsistent with Sullivan ${ }^{[20]}$ who mentioned that about $80 \%$ of nurses in his study were familiar with teach-back method but only $36 \%$ of them used it during the discharge planning.

These findings indicated the nurses' needs for more training programs on implementing comprehensive discharge the teach-back teaching strategies such as teach-back method in order to improve patient understanding of discharge instruction and thus improve their health outcome.

Regarding mother's comprehension of discharge instructions, the current study illustrated that more than half of mothers (65\%) were unable to describe the discharge instructions clearly at pre-intervention phase. These findings were consistent with Miller et al. ${ }^{[24]}$ who found that only $40 \%$ of the patients were able to describe their medication upon discharge. Indeed, another study has shown that $40 \%-80 \%$ of the medical instructions patients are told, is forgotten immediately and about half of that instructions are retained incorrectly. ${ }^{[25]}$ This finding reflects the mothers' needs for a more effective method to be utilized in delivering the discharge instructions.

Moreover, the findings of the current study revealed that the majority of mothers (96.6\%) of the experimental group were able to describe the discharge instructions clearly at post-intervention phase. Consistently with Miller et al. ${ }^{[23]}$ who stated that utilizing teach back method had improved

Published by Sciedu Press patients' understanding of their discharge instruction. They reported that $83.3 \%$ of patient understood their medication.

Moreover, Kripalani et al. ${ }^{[26]}$ demonstrated that teach-back method was an effective method to evaluate understanding of informed consent. These findings reflect the effectiveness of using teach-back method for confirming mothers' understanding of discharge instructions. Furthermore, the Agency for Healthcare Research and Quality had proven the effectiveness of teach-back method to improve and confirm patients' comprehension of discharge instruction.

Regarding self-efficacy, the current study reported that selfefficacy of mothers in the experimental group improved at post-intervention and follow up phases $(57.1 \pm 10.3,52.4$ $\pm 8.4)$. There were high statistical significant differences between experimental, control groups at post-intervention and follow up phase. These findings supported by Edraki et al. ${ }^{[22]}$ who evaluate the effect of an educational program on the quality of life and self-efficacy of the mothers of the infants with congenital heart disease. They confirmed the effectiveness of the educational program on mothers' selfefficacy.

At the same line, Ping ${ }^{[27]}$ mentioned that during the teachback process, patient's transition from having their disease managed by nurses to manage themselves that assumes an increase in patient's capabilities and confidence. In other words, teach-back method increases one's self-efficacy. Otherwise, regardless of patients' health literacy abilities, teachback method had been proved to improve self-efficacy. ${ }^{[28]}$

Regarding mother's level of satisfaction, results of the present study clarified that more than half of mothers in the experimental group were very satisfied $(60 \%)$ and about one quarter $(26.7 \%)$ were slightly satisfied at post-intervention phase. There was a statistical significant difference between mothers at pre- and post-intervention phase regarding their satisfaction with teach-back method.

Consistently with Schillinger et al. ${ }^{[29]}$ who reported that patient satisfaction improved significantly after conducting teach-back method. At the same line, Al-Abri and AlBalushi $^{[30]}$ indicated that effective and clear explanation experienced in the teach-back method, had the strongest impact on improving the overall patient satisfaction. Moreover, they mentioned the importance of nursing role as the most significant determinant of overall patient satisfaction.

The findings of the current study supported the hypothesis of the study that mothers of children with CHD who received discharge instruction through teach-back method had more self-efficacy and satisfaction than mothers who received discharge instruction through regular method. 


\section{Recommendation}

(1) Nurses should incorporate teach-back method as an effective teaching strategy during the discharge planning for children with congenital heart disease.

(2) Ongoing in-service training program about teach-back method should be implemented at all pediatric units to improve nurses' knowledge and skills in discharge planning.

\section{Conclusions}

The current study concluded that most of the nurses were unfamiliar with and did not apply teach-back method in discharge planning. Utilization of teach-back method in discharge instruction improved self-efficacy and satisfaction of mothers' caring for children with congenital heart disease.

\section{CONFLICTS OF INTEREST DisClOSURE}

The authors declare that there is no conflict of interest.

\section{REFERENCES}

[1] Gerdes M, Flynn T. Clinical assessment of neurobehavioral outcomes in infants and children with congenital heart disease. Progress in Pediatric Cardiology. 2010; 29: 97-105. https ://doi .org/10.101 6/j.ppedcard.2010.06.009

[2] National Heart, Lung, and Blood Institute. What Are Congenital Heart Defects? 2010. Availabel from: https ://www.nhlbi.nih. gov/health/health-topics/topics/chd/signs

[3] Hoffman J. Essential Cardiology: Principles and Practice. Totowa, NJ: Humana Press; 2005.

[4] Milunsky A. Genetic Disorders and the Fetus: Diagnosis, Prevention and Treatment. John Wiley \& Sons; 2011.

[5] Mendis S, Puska P, Norrving B, et al. Global Atlas on Cardiovascular Disease Prevention and Control (PDF). World Health Organization in collaboration with the World Heart Federation and the World Stroke Organization. 2011.

[6] Dean SV, Lassi ZS, Imam AM, et al. Preconception care: nutritional risks and interventions. Reproductive Health. 2014; 11(Suppl 3): S3

[7] Mayoclinic. Congenital Heart Defects in Children. 2017. Available from: http://www.mayoclinic.org/diseases-conditions/

[8] Nousi D, Christou A. Factors affecting the quality of life in children with congenital heart disease. Health Science Journal. 2010; 4 94-100.

[9] Berarducci A, Lengacher CA. Self-efficacy: an essential component of advanced-practice nursing. Nursingconnections. 1998 Spring; 11(1): 55-67.

[10] Arafa MA, Zaher SR, El-Dowaty AA, et al. Quality of life among parents of children with heart disease. Health and Quality of Life Outcomes. 2008; 6: 91. PMid:18980676 https ://doi.org/10.1 186/1477-7525-6-91

[11] American Medical Association. Teach-back: Interactive communication loop in patient education. Arch Intern Med. 2003 Jan 13; 153(1): 83-90.

[12] Milford AC. Care of the Family of an Infant With a Congenital Heart Defect During the NICU Hospitalization. J Perinat Neonat Nurs 2016; 30(1): 64-67. PMid:26813393 https://doi.org/10.109 7/JPN. 0000000000000155

[13] Brach C, Keller D, Hernandez LM, et al. Institute of Medicine of the National Academy of Sciences. Washington DC: The National Academies Press; Ten attributes of health literate health care organizations. 2012. 1-26 p.

[14] DeWalt DA, Callahan LF, Hawk VH, et al. Health literacy universal precautions toolkit. Rockville, MD: Agency for Healthcare Research and Quality, Department of Health and Human Services; 2010. Available from: https://www.ahrq.gov/professionals/qua lity-patient-safety/quality-resources/tools/liter acy-toolkit/index.html

[15] Dekker J, Van Dijk GM, Veenhof C. Risk factors for functional decline in osteoarthritis of the hip or knee. Current Opinion in Rheuma- tology. 2009; 21: 520-524. PMid:19550331 https ://doi.org/10 .1097/BOR . 0b013e32832e6eaa

[16] Davis GC. Improved sleep may reduce arthritis pain. Holistic Nursing Practice. 2003; 17: 128-135. PMid:12784896 https : //doi .org/ 10.1097/00004650-200305000-00003

[17] Dirik G, Karanci AN. Psychological distress in rheumatoid arthritis patients: An evaluation within the conservation of resources theory. Psychology \& Health. 2009; 25(5): 617-632.

[18] Mark R. Self-efficacy and arthritis disability: An updated synthesis of the evidence base and its relevance to optimal patient care. Health Psychol Open. 2014 Jul; 1(1). https ://doi.org/10.1177/2055 102914564582

[19] Sherer M, Madox EJ. The self-effacicy scale: constriction and validation. Psychological Reports. 1982; 51: 663-671. https ://doi.or g/10.2466/pr0.1982.51.2.663

[20] Sullivan O, Kathleen. Improving patient outcomes through use of the teach-back method in the Post Anesthesia Care Unit. Master' s Projects. 2014.

[21] Lippitt R, Watson J, Westley B. The Dynamics of Planned Change (New York, Harcourt Brace, 1958). In: Kozier B, Erb G, Berman A and Burke K. Fundamentals of Nursing: Concepts, Process, and Practice. (6th ed.) Prentice Hall Health. New Jersey. 2000.

[22] Edraki M, Kamali M, Beheshtipour N, et al. Int J Community Based Nurs Midwifery. 2014 Jan; 2(1): 51-59.

[23] Wong DL, Hockenberrry ME, Winkelstein ML, et al. Whaley and Wong's Nursing Care of Infants and Children. 8th ed. Mosby; 2011.

[24] Shelley M, Lattanzio M, Cohen S. Teach-back from a patient's perspective. Nursing. 2016; 46(2): 63-64.

[25] Agency of Health Care Research Quality. CAHPS surveys and tools to advance patient-centered care: Medication communication. 2015 Available from: https://cahps.ahrq.gov/surveysguidance /hospital/hcahps_slide_sets/medication_communicati on/medication_communication.html

[26] Kripalani S, Bengtzen R, Henderson L, et al. Clinical research in lowliteracy populations: using teach-back to assess comprehension of informed consent and privacy information. IRB: Ethics \& Human Research. 2008; 30(2): 13-19.

[27] Xu P. Using teach-back for patient education and self-management. 2012.

[28] Weiss BD. Health literacy and patient safety: Help patients understand. 2nd ed. American Medical Association Foundation and American Medical Association. 2007.

[29] Schillinger D, Piette J, Grumbach K, et al. Closing the loop: physician communication with diabetic patients who have low health literacy. Archives of Internal Medicine. 2003; 163(1): 83-90.

[30] Al-Abri R, Al-Balushi A. Patient satisfaction surveys as a tool towards quality improvement. Oman Medicine Journal. 2014; 29(1): 3-7. Available from: http://www.ncbi.nlm.nih.gov/pmc/art icles/PMC3910415/ 